in vivo $35:$ 693-697 (2021)

doi:10.21873/invivo.12309

Review

\title{
Monomeric C-Reactive Protein - A Feature of Inflammatory Disease Associated With Cardiovascular Pathophysiological Complications?
}

\author{
YASMIN ZEINOLABEDINY, SHANT KUMAR and MARK SLEVIN \\ Department of Life Sciences, Metropolitan University, Manchester, Manchester, U.K.
}

\begin{abstract}
Monomeric $C$-reactive protein $(m C R P)$, the dissociated form of native $C$-reactive protein, is a critical molecule that causes and perpetuates inflammation in serious diseases. It has 'adhesive'-like properties causing aggregation of blood cells and platelets, and can stick permanently within arterial tissue where it can contribute to further complications including thrombosis, linking it potentially to atherosclerosis and subsequent acute coronary events. In this mini review, we discuss briefly the implications and the potential value of measuring and manipulating it for clinical diagnostics and therapeutic purposes.
\end{abstract}

C-reactive protein (CRP) is a an acute-phase pentraxin produced by the liver, usually during/following infection or tissue trauma in large quantities (up to 1,000-fold increase) compared to normal, systemic circulating levels, and released into the blood directly in response to macrophage, adipocyte or T-cell release of IL-6. It circulates as soluble native CRP (nCRP), which is relatively biologically inactive, and whose main functions are to mediate complement activation and bind to foreign or damaged cells thereby, promoting phagocytosis (1). It was first shown almost 30 years ago, that nCRP could dissociate into 5 identical sub-units of monomeric C-reactive protein (mCRP), which had strikingly different biological properties to the parent molecule (2). The astonishing properties of only the mCRP have been highlighted over the last decade by the explosion in publications year upon year to 2020 .

This article is freely accessible online.

Correspondence to: Mark Slevin, School of Life Sciences, Faculty of Science and Engineering, Manchester Metropolitan University, Manchester, U.K. Tel: +44 1612471172, e-mail: m.a.slevin@mmu.ac.uk

Key Words: Cardiovascular, monomeric c-reactive protein, inflammation, diagnostic, therapeutic, review.
CRP dissociates into mCRP directly upon contact with activated tissue, or cells (including circulating monocytes and platelets), particularly at sites of inflammation or infection (3). The mechanism through which cells, activated platelets or liposomes induce dissociation of CRP involves its binding to cell surface phosphocholine or lyso-phosphatidylcholine, followed by interaction with specific receptors at membrane lipid-rafts (e.g. Fc $\gamma \mathrm{RI}$ and II in phagocytic cells) through the mCRP-cholesterol-binding region (aa35-47) (4 and references there in). Next, mCRP stimulates enhanced local production of pro-inflammatory cytokines including IL-8, MCP-1, TNF$\alpha$ IL-6, and associated transcription factors such as Nf-kB (3).

\section{mCRP and Cardiovascular Disease}

mCRP is a critical molecule that causes and perpetuates inflammation based on its ability to cause aggregation of blood cells linked to onset of acute coronary disease and atherosclerotic thrombotic plaques $(5,6)$. It can stick within the walls/intima of major arteries where it contributes significantly to worsening of atherosclerosis and the formation of unstable plaques increasing the likelihood of rupture and concomitant heart attack or stroke (7). Total native CRP levels in the blood correlate strongly with the above and clinical symptoms of unstable angina, myocardial infarction, stroke and peripheral artery disease (8).

mCRP contributes significantly to thrombosis, while pentameric nCRP has no involvement in thrombogenesis. Specifically, blood mCRP induces platelet activation and thrombus growth (1). It has been shown that mCRP causes platelet aggregation and recruitment under high flow/sheer stress conditions $(9,10)$, and trapped mCRP inside growing platelet aggregates stimulated further platelet deposition (11). It has also been shown that mCRP induced increased fibrin polymerization in association with tissue factor expression; both strongly associated with the pro-coagulant activity of monocytes, clot production and thrombosis (12). 


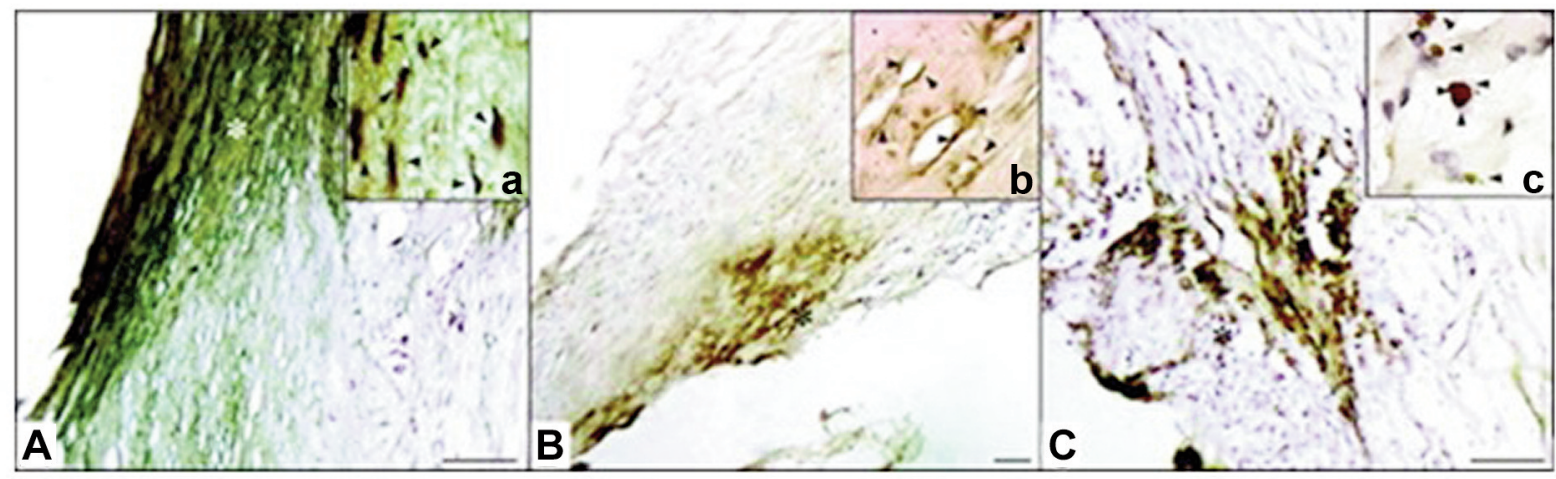

Figure 1. Unstable atherosclerotic plaques showing strong neo-vessel rich staining for mCRP (high power inserts; coloured brown-HRP). Our previous work - (13).

\section{CRP and Atherosclerotic Plaque Development}

CRP contributes significantly to cardiovascular disease progression through cellular activation and ligand binding, primarily, to the fragment crystallizable region gamma receptors (Fc $\gamma \mathrm{Rs}) . \mathrm{mCRP}$ is not present in the walls of healthy vessels arteries or in other tissues, but it becomes detectable in the early stages of atherogenesis and accumulates during the progression of atherosclerosis in association with increasing inflammation (13; Figure 1). In addition, mCRP accumulates within the infarcted myocardium after a heart attack and circulating mCRP levels are indicative of the severity of the disease and mortality (14).

Only mCRP is biologically active. It is present in the necrotic core of plaques, inhibits endothelial nitric oxide production and increases reactive oxygen species generation, contributing to atherosclerotic plaque instability by a variety of mechanisms. These include, increasing endothelial cell adhesiveness through the expression adhesion molecules, promotion of leukocyte and monocyte recruitment into the atheromatous plaque (promoting inflammation), enhancement of lipid accumulation, induction of cellular apoptosis and matrix break-down (e.g. through matrix metalloproteinase production), and by enzymatically binding to modified lowdensity lipoprotein (7, 15 and references therein). Recently, Ullah et al. (16), showed that blood mCRP produced in the local circulation binds with high affinity to fibronectin, significantly enhancing the adhesion of monocytes and promoting the inflammatory response.

\section{mCRP and Vasculogenesis Contribution to the Disease}

In addition, mCRP has significant pro-angiogenic and remodelling effects that can promote progression from a stable arterial thickening to an unstable tissue through rapid expansion as a result of the aberrant permeability of angiogenic blood vessels 'that associates with structural changes, increased inflammation and instability of the atherosclerotic plaque (7, 9). mCRP has been shown to promote aberrant angiogenesis with leaky microvessels in the plaque core contributing to weakening and instability through haemorrhage and leukocyte and macrophage deposition (9). The capacity of mCRP to induce haemorrhagic vascularization in tissue and create permeable endothelial monolayer gap-junctions has been shown in vivo by our group (17) (Figure 2).

\section{CRP-mCRP Detection and Modulation as a Novel Diagnostic, Prognostic and Therapeutic}

The studies presented above suggest a possible novel therapeutic (protective, preventative) mechanism by blocking the conversion of nCRP (pentameric) to mCRP.

Our studies have shown that small off-target orphan molecules, particularly acetylcholine, due to its similarities with phosphocholine, demonstrated probable interaction with and binding to mCRP-phosphatidylcholine, blocked endothelial-monocyte activation and platelet aggregation, thereby nullifying inflammation (IL-6/TNF- $\alpha$ ) in vitro (4). Regarding nCRP-dissociation inhibition, compounds containing phosphocholine dimers (five molecules between two pentamers) can potentially cross-link two CRP molecules together preventing dissociation into monomers, and has been shown to block CRP binding to LDL and inhibit $\mathrm{C} 1 \mathrm{q}$ complement activation (18). Autoantibodies to mCRP (aa3547) generated by patients with lupus nephritis inhibited the activity of complement factor $\mathrm{H}$ and predicted the most severe renal damage and worse outcome (19). Hence, providing a possible therapeutic and diagnostic target for auto-immune diseases. In addition, recently, Singh and Argrawal (20), described an mCRP mutant that was atheroprotective in a murine model of atherosclerosis providing an opportunity to 

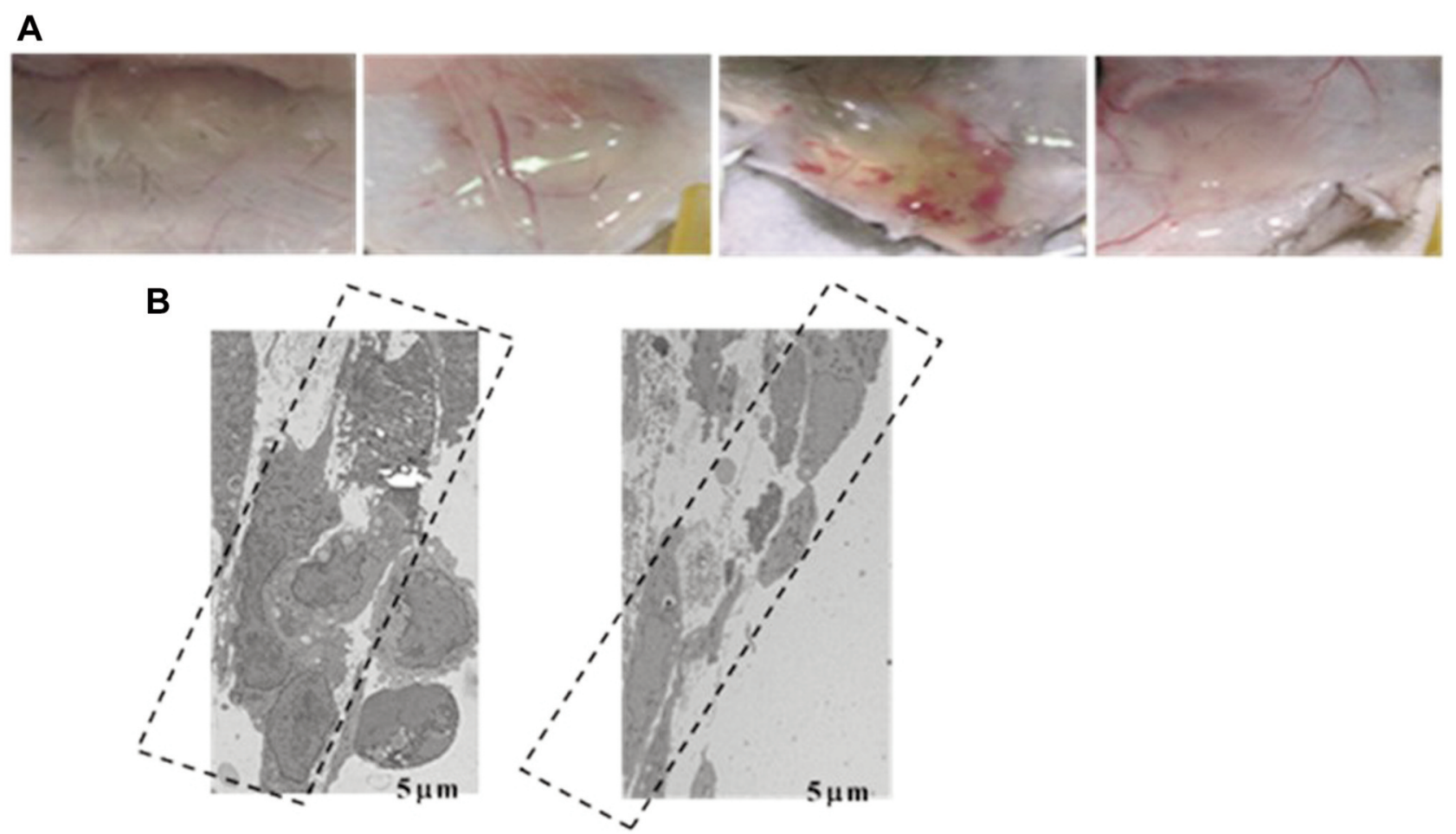

C

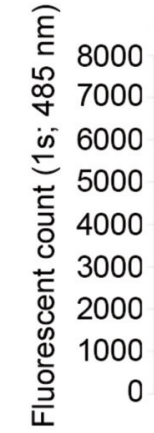

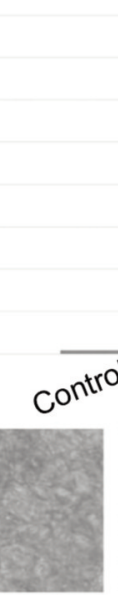

Control

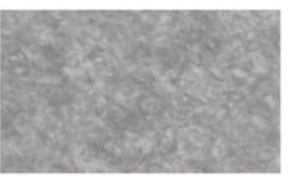

Control

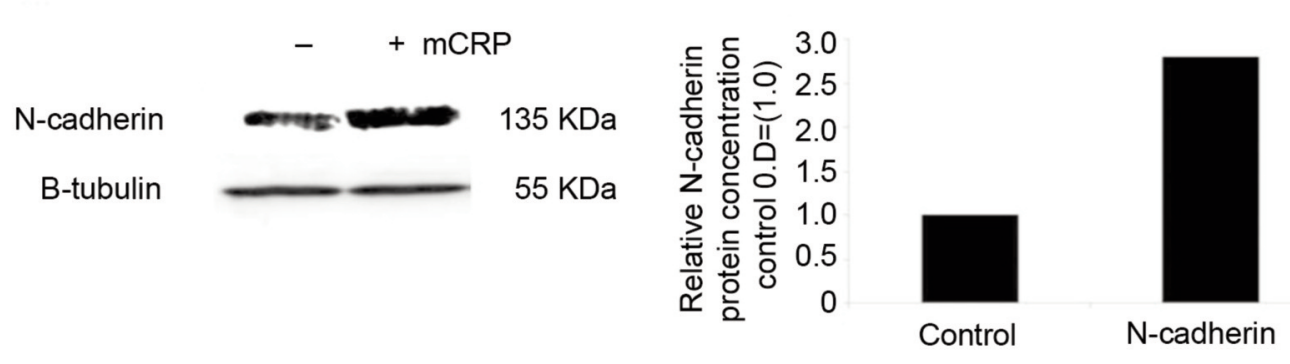

D

Figure 2. $m C R P$ effects on vasculature permeability and patency. A) (top left to right) shows the effect of murine sub-cutaneous injection of PBS, $n C R P$, $m C R P$ and VEGF in matrigel after 1 week, note the vascular aberrant haemorrhagic angiogenesis in the $m C R P$-containing sample. B) (left) normal TEM of gap junctions in aortic endothelial cells and (right)after treatment with $m C R P$, note degradation at the site creating increased permeability and potential inflammation. C) (top/middle images) enhanced permeability of mCRP-treated endothelial monolayer cultures and FITC-labelled dextran (lower)-increased expression of $N$-cadherin (western blotting) by the endothelial cells after mCRP treatment-a marker of adhesion and activation (16). 
create a novel therapeutic that when bound to LDL would reduce foam cell formation and local inflammation.

In terms of the measurement and detection of mCRP as a potential prognostic indicator of acute coronary events, $\mathrm{mCRP}$ may be a marker specific to the underlying pathological processes. Indeed, circulating or microparticle-bound mCRP (or bound to platelets/macrophages etc.) is a far better diagnostic index than nCRP in identifying and profiling myocardial infarction and peripheral artery disease as well as prognosis and follow up of stroke and inflammatory conditions including Lupus and giant cell arteritis. Development of an ELISA that discriminates nCRP from mCRP could provide a far more accurate method for the prediction of acute risk in acute myocardial infarction patients.

Crawford et al. (6) showed that the high-sensitivity CRP assay (hsCRP) did not measure pentameric CRP or mCRP on microparticles (MPs), whereas flow cytometry did. MPs derived from endothelial cells, particularly those bearing mCRP, were elevated in peripheral artery disease (PAD) patients compared to controls. The numbers of mCRP (+) endothelial MPs did not correlate with hsCRP measurements of soluble pCRP, indicating their independent modulation. In a model of vascular inflammation, mCRP induced endothelial shedding of MPs and was proinflammatory, while pCRP was anti-inflammatory. mCRP on endothelial MPs may be both an unmeasured indicator and an amplifier of vascular disease, and its detection might improve risk sensitivity. Melnikov et al. (21) also showed the significantly increased capacity of exosomes and microparticles derived from monocytes to transport mCRP in the blood from patients with coronary artery disease.

Currently, on the market, there is no successful assay to directly measure plasma bound mCRP. However, recently, Zhang et al. described using antibodies although not specific for mCRP, did not bind to the native form of mCRP at low concentrations (22). They successfully used this experimental kit to show that mCRP was a significantly better marker than nCRP in determining inflammatory status of several skin-related autoimmune disorders.

\section{Conclusion}

To summarise, there is now significant persuasive evidence implicating $\mathrm{mCRP}$ as a significant contributor to cardiovascular disease, creating a positive microenvironment for the perpetuation of inflammation within plaques, and promoting endothelial dysfunction, complement binding, monocyte polarization and platelet aggregation (23). Therefore, there should be a focussed effort to provide new diagnostic methods to measure its circulating levels in relation to diseases and to consider therapeutics that can abate its action within the tissues if not prevent the initial dissociation during acute inflammatory situations.

\section{Conflicts of Interest}

There are no conflicts of interest to declare regarding this study.

\section{Authors' Contributions}

YZ-drafted the manuscript, SK and MS made the literature search and provided the figures.

\section{Acknowledgements}

This work was supported from a grant from the Competitiveness Operational programme 2014-2020: C-reactive protein therapy for stroke-associated dementia: ID_P_37_674, My SMIS code:103432 contract 51/05.09.2016.

\section{References}

1 McFadyen JD, Kiefer J, Braig D, Loseff-Silver J, Potempa LA, Eisenhardt SU and Peter K: Dissociation of C-reactive protein localizes and amplifies inflammation: evidence for a direct biological role of C-Reactive protein and its conformational changes. Front Immunol 12(10): 1351, 2018. PMID: 29946323. DOI: $10.3389 /$ fimmu.2018.01351

2 Polevshikov AV, Nazarov PG and Berestovaia LK: The effect of C-reactive protein on RNA synthesis and neutrophil proteins. Vopr Med Khim 39(1): 43-45, 1993. PMID: 7684545.

3 Sproston NR and Ashworth JJ: Role of C-reactive protein at sites of inflammation and infection. Front Immunol 13(9): 754, 2018. PMID: 29706967. DOI: 10.3389/fimmu.2018.00754

4 Slevin M, Iemma RS, Zeinolabediny Y, Liu D, Ferris G R, Caprio V, Phillips N, Di Napoli M, Guo B, Zeng X, AlBaradie R, Binsaleh N K, McDowell G and Fang W: Acetylcholine inhibits monomeric $\mathrm{C}$-reactive protein induced inflammation, endothelial cell adhesion, and platelet aggregation; A potential therapeutic? Front Immunol 26(9): 2124, 2018. PMID: 30319609. DOI: $10.1038 /$ srep13281

5 Thiele JR, Habersberger J, Braig D, Schmidt Y, Goerendt K, Maurer V, Bannasch H, Scheichl A, Woollard KJ, von Dobschütz E, Kolodgie F, Virmani R, Stark GB, Peter K and Eisenhardt SU: Dissociation of pentameric to monomeric c-reactive protein localizes and aggravates inflammation: in vivo proof of a powerful proinflammatory mechanism and a new antiinflammatory strategy. Circulation 130(1): 35-50, 2014. PMID: 24982116. DOI: 10.1161/CIRCULATIONAHA.113.007124

6 Crawford JR, Trial J, Nambi V, Hoogeveen RC, Taffet GE and Entman ML: Plasma levels of endothelial microparticles bearing monomeric C-reactive protein are increased in peripheral artery disease. J Cardiovasc Transl Res 9(3): 184-193, 2016. PMID: 26891844. DOI: $10.1007 / \mathrm{s} 12265-016-9678-0$

7 Badimon L, Peña E, Arderiu G, Padró T, Slevin M, Vilahur G and Chiva-Blanch G: C-reactive protein in atherothrombosis and angiogenesis. Front Immunol 2(9): 430, 2018. PMID: 29552019. DOI: $10.3389 /$ fimmu.2018.00430

8 Boncler M, Wu Y and Watala C: The multiple faces of Creactive protein-physiological and pathophysiological implications in cardiovascular disease. Molecules 24(11): 2062, 2019. PMID: 31151201. DOI: 10.3390/molecules 24112062 
9 Slevin M and Krupinski J: A role for monomeric C-reactive protein in regulation of angiogenesis, endothelial cell inflammation and thrombus formation in cardiovascular/cerebrovascular disease? Histol Histopathol 24(11): 1473-1478, 2009. PMID: 19760596. DOI: $10.14670 / \mathrm{HH}-24.1473$

10 Molins B, Peña E, de la Torre R and Badimon L: Monomeric Creactive protein is prothrombotic and dissociates from circulating pentameric C-reactive protein on adhered activated platelets under flow. Cardiovasc Res 92(2): 328-337, 2011. PMID: 21859817. DOI: $10.1093 / \mathrm{cvr} / \mathrm{cvr} 226$

11 De la Torre R, Peña E, Vilahur G, Slevin M and Badimon L: Monomerization of C-reactive protein requires glycoprotein IIbIIIa activation: pentraxins and platelet deposition. J Thromb Haemost 11(11): 2048-2058, 2013. PMID: 24119011. DOI: $10.1111 /$ jth. 12415

12 Li R, Ren M, Luo M, Chen N, Zhang Z, Luo B and Wu J: Monomeric C-reactive protein alters fibrin clot properties on endothelial cells. Thromb Res 29(5): e251-256, 2012. PMID: 22475312. DOI: 10.1016/j.thromres.2012.03.014

13 Krupinski J, Turu MM, Martinez-Gonzalez JM, Carvajal A, Juan-Babot JO, Iborra E, Slevin M, Rubo F and Badimon L: Endogenous expression of C-reactive protein is increased in active (ulcerated noncomplicated) human carotid artery plaques. Stroke 37(5): 1200-1204, 2006. PMID: 16601222. DOI: 10.1161/01.STR.0000217386.37107

14 Wang J, Tang B, Liu X, Wu X, Wang H, Xu D and Guo Y: Increased monomeric CRP levels in acute myocardial infarction: a possible new and specific biomarker for diagnosis and severity assessment of disease. Atherosclerosis 239(2): 343-349. 2015. PMID: 25682033. DOI: 10.1016/j.atherosclerosis.2015.01.024

15 Thiele JR, Zeller J, Kiefer J, Braig D, Kreuzaler S, Lenz Y, Potempa LA, Grahammer F, Huber TB, Huber-Lang M, Bannasch H, Stark GB, Peter K and Eisenhardt SU: A conformational change in $\mathrm{C}$-reactive protein enhances leukocyte recruitment and reactive oxygen species generation in ischemia/reperfusion injury. Front Immunol 16(9): 675, 2018. PMID: 29713320. DOI: 10.3389/fimmu.2018.00675

16 Ullah N, Ma FR, Han J, Liu XL, Fu Y, Liu YT, Liang YL, Ouyang $\mathrm{H}$ and $\mathrm{Li} \mathrm{HY}$ : Monomeric C-reactive protein regulates fibronectin mediated monocyte adhesion. Mol Immunol 117: 122-130, 2020. PMID: 32622210. DOI: 10.1016/j.molimm.2019.10.013
17 Boras E, Slevin M, Alexander MY, Aljohi A, Gilmore W, Ashworth J, Krupinski J, Potempa LA, Al Abdulkareem I, Elobeid A and Matou-Nasri S: Monomeric C-reactive protein and Notch-3 co-operatively increase angiogenesis through PI3K signalling pathway. Cytokine 69(2): 165-179, 2014. PMID: 24972386. DOI: 10.1016/j.cyto.2014.05.027

18 Caprio V, Badimon L, Di Napoli M, Fang WH, Ferris GR, Guo B, Iemma RS, Liu D, Zeinolabediny $Y$ and Slevin M: pCRP-mCRP dissociation mechanisms as potential targets for the development of small-molecule anti-inflammatory chemotherapeutics. Front Immunol 28(9): 1089, 2018. PMID: 29892284. DOI: 10.3389/ fimmu.2018.01089

19 Li QY, Li HY, Yu F, Wu Y and Zhao MH: Autoantibodies against C-reactive protein influence complement activation and clinical course in lupus nephritis. J Am Soc Nephrol 28(10): 3044-3054, 2017. PMID: 28566480. DOI: 10.1681/ASN.2016070735

20 Singh SK and Agrawal A: Functionality of C-reactive protein for atheroprotection. Front Imunol 16(10): 1655, 2019. PMID: 31379851. DOI: 10.3389/fimmu.2019.01655

21 Melnikov I, Kozlov S, Saburova O, Zubkova E, Guseva O, Domogatsky S, Arafieva T, Radyukhina N, Zvereva M, Avtaeva Y, Buryachkovskaya L and Gabbasov Z: CRP is transported by monocytes and monocyte-derived exosomes in the blood of patients with coronary artery disease. Biomedicines $8(10): 435$, 2020. PMID: 33086769. DOI: 10.3390/biomedicines8100435

22 Zhang L, Li HY, Li W, Shen ZY, Wang YD, Ji SR and Wu Y: An ELISA assay for quantifying monomeric c-reactive protein in plasma. Front Immunol 12(9): 511, 2018. PMID: 29593741. DOI: $10.3389 /$ fimmu.2018.00511

23 Melinikov IS, Kozlov SG, Saburova OS, Avtaeva YN, Prokofieva LV and Gabbasov ZA: Current position on the role of monomeric C-reactive protein in vascular pathology and atherothrombosis. Curr Pharm Des 36(1): 37-43, 2020. PMID: 31840602. DOI: $10.2174 / 1381612825666191216144055$

Received November 18, 2020

Revised December 15, 2020

Accepted December 17, 2020 